\title{
Impact of Oil-Based Drilling Fluids Emulsification on Tolerance and Hydrocarbon-Degrading Potential of Ralstonia pickettii and Alcaligenes piechandii
}

\author{
Victoria Kostenko ${ }^{1,3 *}$, Robert John Martinuzzi ${ }^{2}$ and Geir Hareland ${ }^{3}$ \\ ${ }^{1}$ Calgary Center for Innovative Technology, University of Calgary, University Drive NW, Calgary, Alberta, Canada \\ ${ }^{2}$ Mechanical and Manufacturing Engineering Department, University of Calgary, University Drive NW, Calgary, Alberta, Canada \\ ${ }^{3}$ Chemical and Petroleum Engineering Department, University of Calgary, University Drive NW, Calgary, Alberta, Canada
}

\begin{abstract}
Biodegradability of drilling fluid waste is essential in the development of environmentally compatible oil and gas drilling. Biodegradation is determined not only by the enzymatic potential, but also microbial tolerance to hydrocarbons. The present study investigated the hydrocarbon degradation and tolerance of Ralstonia pickettii BP20 and Alcaligenes piechandii KN1 to drilling fluids with diesel and low-aromatic continuous phases in respect to their state: non-emulsified oil, direct $(\mathrm{O} / \mathrm{W})$ and invert $(\mathrm{W} / \mathrm{O})$ emulsions; and the concentrations affecting microbial activity and viability. In general, $A$. piechaudii KN1demonstrated higher tolerance than $R$. picketti BP20 did; but, the impacts of different drilling fluids on viability and activity of both microbial strains had similar trends. Microbial growth and hydrocarbons degradation rates increased when diesel was replaced with low aromatic oil, and emulsified The higher productivity was observed in direct $(\mathrm{O} / \mathrm{W})$ emulsions than in invert $(\mathrm{W} / \mathrm{O})$ emulsions. Similarly, viability of microorganisms in low aromatic fluids and emulsions was higher than in corresponding diesel drilling fluids. Tolerance to low aromatic fluids increased in the order: non-emulsifier oil < invert emulsion < direct emulsion. In contrast, for diesel based drilling fluids, direct emulsion enhanced, but invert emulsion reduced microbial viability compared to non-emulsified oil.
\end{abstract}

Keywords: Hydrocarbon-degrading Microorganisms; Tolerance; Drilling fluid; Low-aromatic; Hydrocarbons; Emulsion

Abbreviations: DF - Diesel-based Fluid; DIE - Diesel-Based Invert
Emulsion; DDE - Diesel-Based Direct Emulsion; LAF - Low Aromatic
Drilling Fluid; LAIE - Low Aromatic Invert Emulsion; LADE - Low
Aromatic Direct Emulsion; O/W - oil in water emulsions; W/O - water
in oil emulsions; WBF- Water-based Drilling Fluid; NADF - Non-
Aqueous Drilling Fluid; IEDF - Invert Emulsion Drilling Fluid; PAH
- Polyaromatic Hydrocarbons; TPH - Total Petroleum Hydrocarbons;
HLB - Hydrophilic-Lipophilic Balance; MBC - Minimum Bactericidal
Concentration; MIC - Minimum Inhibitory Concentration; MSC -
Minimum Suppressive Concentration; Cp - Peak Concentration; CFU
- Colony Forming Units

\section{Introduction}

Drilling fluids are essential for the drilling process in the oil and gas industry. Drilling fluids are used to control formation pressure, lubricate and cool the drilling bit, remove cuttings from the drilling well, and form a consolidated filter cake on the sides of the hole to prevent loss circulation. There are three major types of drilling fluids: WaterBased Fluids (WBF), non-aqueous drilling fluids (NADF with diesel, mineral or synthetic oil) and Invert Emulsion Drilling Fluids (IEDF). Drilling operated with WBF suffer some operating limitations. In many cases, NADF and IEDF replace WBF to improve drilling performance with respect to higher rate of penetration, more lubrication of the drill string, stability of the sensitive and highly reactive formation, and rheological stability of the drilling fluid under high pressure and high temperature drilling conditions.

While improving the performance characteristics is a ubiquitous priority in drilling operation development, the environmental acceptability of drilling fluids and associated waste is a more recent but rapidly growing concern. The negative effect of drilling fluids and waste on human and environmental health is attributed to their toxicity and poor biodegradability. Reduced toxicity and enhanced biodegradability of drilling fluids have been reported when diesel was replaced with mineral and synthetic oils $[1,2]$. Diesel contains high concentrations of Polyaromatic Hydrocarbons (PAHs) and their derivatives; while mineral and synthetic oils are low- or non-aromatic compounds. Microorganisms have the capacity to degrade the majority of natural hydrocarbons including alkanes, monoaromatic and lowmolecular-weight PAHs. The higher-molecular-weight PAHs are more toxic and recalcitrant to biodegradation [3]. Microbial degradation of recalcitrant compounds may be improved via emulsification and solubilization in the aqueous phase, which may be achieved through the action of surfactants [4-6]. Since hydrocarbon oxidation is performed by intracellular enzymes, the solubilization of hydrocarbons enhances the mass transfer rate of hydrocarbons to water phase and into microbial cells, and hence, increases the degradation rate. Additionally, emulsification increases bioavailability of hydrocarbons, and hence biodegradation rate, due to increased oil-water interface available for microbial growth [7]. Thus, surfactant addition and emulsification of oil have a marked impact on hydrocarbon degradation. However, studies on the bioremediation of hydrocarbons by microorganisms observed that these compounds impaired the microbial growth and

*Corresponding author: Victoria Kostenko, Calgary Center for Innovative Technology, University Drive NW, University of Calgary, Calgary AB, Canada T2N 1N4, Tel: +1-403-210-7592; E-mail: vkostenk@ucalgary.ca

Received April 11, 2013; Accepted May 24, 2013; Published May 31, 2013

Citation: Kostenko V, Martinuzzi RJ, Hareland G (2013) Impact of Oil-Based Drilling Fluids Emulsification on Tolerance and Hydrocarbon-Degrading Potential of Ralstonia pickettii and Alcaligenes piechandii. J Pet Environ Biotechnol 4: 140 doi:10.4172/2161-1459.1000140

Copyright: (C 2013 Kostenko V, et al. This is an open-access article distributed under the terms of the Creative Commons Attribution License, which permits unrestricted use, distribution, and reproduction in any medium, provided the original author and source are credited. 
hydrocarbon degradation at relative low hydrocarbon concentrations, and hydrocarbon solubilization further enhanced the antimicrobial action of petroleum compounds [8-11].

The effect of the hydrocarbon concentration on biodegradation efficiency has been recently considered. The diesel assimilation by microorganisms decreased as diesel concentration increased from $0.2 \%$ to $1 \%$, and completely stopped above this level [12]. The solubilization of diesel PAHs via derivatization (phenolic or quinonic) or cosolvent addition further reduced acceptable (non-toxic) hydrocarbon concentrations $[8,13]$. The mechanisms of hydrocarbon toxicity against microorganisms involves partitioning of the hydrocarbons into the cytoplasmic membrane, which alters: energy transduction; nutrient and enzyme transfer across the biological membranes; and damages the cytoplasmic membrane which leads to leakage of macromolecules (RNA, proteins, etc), and hence cell death $[8,14]$. The partitioning of the hydrocarbons into the cytoplasmic membrane, similar to the hydrocarbon transport into the cells required for degradation reactions, is a function of hydrocarbon concentration and solubilization. Thus, the concentrations of hydrocarbons, both actual and solubilized in aqueous phase, determine the response of the microorganisms to the hydrocarbon substrate: the hydrocarbons can serve as nutrients at relatively low concentrations, but inhibit or even kill the degrading microorganism at higher concentrations. This critical hydrocarbon level affecting microbial activity and viability is determined by both the hydrocarbon properties (chemistry and bioavailability) and microbial properties such as biodegrading capacity and tolerance, i.e. a microorganism's ability to grow and degrade in the presence of toxic materials. While biochemical potential and tolerance against certain types of substrate are inherent properties of the microorganism and cannot be changed for a particular strain without genetic manipulation; chemistry and state of substrate can be altered to provide microbial response at a desired level. As mentioned above, toxic effects of the drilling fluid chemistry may be eliminated by replacing the PAH compounds with low aromatic compounds; while the bioavailability may be corrected by oil emulsification resulting in enhanced solubility of hydrocarbons and increased interface area for microbial attachment. The oil-in-water (direct) emulsions are known to improve biodegradation of different types of petroleum hydrocarbons [15]. In contrast, the impact of invert (W/O) emulsions, traditionally used in gas and oil drilling, on biodegradation is poorly investigated. However, it has been observed that microbial cells preferentially grow in the water phase (which is limited by small droplets in invert emulsions) and on the oil-water interface. Moreover, continuous oil phase or undispersed oil cause nutrients and oxygen limitation, and hence can reduce hydrocarbon degradation due to substrate limitation [16]. Another issue is the concentration of oil in emulsions allowing effective hydrocarbon degradation without impairing microbial growth since both direct and invert emulsions can increase toxicity of hydrocarbons through solubilization, and hence reduce tolerance of microbial cells to this substrate. Conversely, high microbial tolerance to drilling fluids allows better waste treatment by reducing the extensive waste dilution required to avoid toxic level of hydrocarbons. Thus, along with the isolation of the hydrocarbon-degrading microorganisms, the biodegradation design should be geared to the isolation of the strains with high inherent tolerance to hydrocarbons and/or setting up incubation conditions (e.g., emulsification of hydrocarbon) facilitating microbial tolerance to high hydrocarbon concentrations.

The present study investigates the tolerance of two hydrocarbondegrading microorganisms Ralstonia pickettii BP20 and Alcaligenes piechandii $\mathrm{KN} 1$ to drilling fluids with diesel and low-aromatic continuous phases in respect to (i) their state: non-emulsified oil, direct and invert emulsions; and (ii) the concentrations affecting productivity and viability of microorganisms. The assessment of the productivity is based on two criteria: maximum microbial growth / hydrocarbon assimilation; and the concentrations of drilling fluids providing the maximum microbial activity. While the first parameter aims at increasing degradation rates; the second provides insights about the substrate concentration maintaining the effective biodegradation process. The viability of microorganisms in response to drilling fluid is assessed by determining the minimum oil concentrations causing microbial growth suppression, complete inhibition and microbial population killing. These parameters are necessary to determine the minimum acceptable (non-toxic) hydrocarbon load for bioremediation purpose. The scope of the investigation is: (i) to identify the microbe-drilling fluid interaction patterns with respect to the concentration of the number of aromatic components and emulsification (direct or invert); (ii) set up diagnostic criteria for assessing microbial tolerance to drilling fluids; and (iii) determine the optimum combination of microorganisms and drilling fluid type to provide better biodegradability of drilling waste.

\section{Materials and Methods}

\section{Microorganisms and incubation media}

Ralstonia pickettii BP20 and Alcaligenes piechandii KN1 enriched from hydrocarbon-contaminated Czechowice-Dziedzice Oil Refinery Waste Lagoon were kindly provided by Savannah River National Laboratory (USA). Microorganisms were incubated in the basic mineral medium containing casein hydrolysate $(0.5 \mathrm{~g} / \mathrm{l})$, peptone $(0.5$ $\mathrm{g} / \mathrm{l})$, yeast extract $(0.5 \mathrm{~g} / \mathrm{l}), \mathrm{K}_{2} \mathrm{HPO}_{4}(0.3 \mathrm{~g} / \mathrm{l}), \mathrm{MgSO}_{4}(0.05 \mathrm{~g} / \mathrm{l}), \mathrm{NH}_{4} \mathrm{Cl}$ $(0.1 \mathrm{~g} / \mathrm{l}), \mathrm{CaCl}_{2}(0.05 \mathrm{~g} / \mathrm{l})$. The basic medium was supplemented with either glucose $(0.5 \mathrm{~g} / \mathrm{l})$ or sodium pyruvate $(0.3 \mathrm{~g} / \mathrm{l})$ and used to prepare challenge media loaded with drilling fluids.

\section{Drilling fluid samples}

Diesel Based Fluid (DF) and corresponding Invert Emulsion (DIE) and Direct Emulsion (DDE); and Neodrill Low Aromatic Based Fluid (LAF) and corresponding Invert Emulsion (LAIE) and Direct Emulsion (LADE) were used in these experiments. Emulsions with oil-to-water ratio of 50:50 were stabilized with $5 \%$ of Span 80 (HLB, 4.3) - Tween 80 (HLB, 15.0) mixture as follows. The surfactant mixture of HLB 6.4 (Span 80-to-Tween 80 ratio of 80:20) was stirred with either diesel or Neodill for $5 \mathrm{~min}$. Then water was added dropwise to oil-surfactant mixture while mixing at $10,000 \mathrm{rpm}$. The surfactant mixture of HLB 12.8 (Span 80-to-Tween 80 ratio of 20:80) was dissolved in water. Then either diesel or Neodill was added dropwise to the water-surfactant solution while mixing at $10,000 \mathrm{rpm}$. The drilling fluid samples were diluted in incubation media to final concentrations of oil ranging from 0.1 to $10 \%(\mathrm{v} / \mathrm{v})$ with two-fold increments. Drilling fluid-free incubation medium was used as control. Challenge and control media contained either glucose or pyruvate as additional carbon sources.

\section{Tolerance of microorganisms to drilling fluids}

Challenge and control media were seeded with microorganism at concentration of $10^{7} \mathrm{cell} / \mathrm{ml}$ and incubated at $25^{\circ} \mathrm{C}$ with agitation at $100 \mathrm{rpm}$ for 24 hours. Microbial suspensions were then serially diluted in phosphate buffer saline and plated on agar basic media with glucose to determine the number of viable cells after treatment. The cell number was represented as $\log \mathrm{CFU} / \mathrm{ml}$ (CFU - colony forming units). Changes in viable cell numbers $(\Delta \log \mathrm{CFU} / \mathrm{ml})$ after 24 hour exposure to challenge and control media were used to determine growth rates (positive $\Delta \log \mathrm{CFU} / \mathrm{ml}$ ) and killing rates (negative $\Delta \log \mathrm{CFU} / \mathrm{ml}$ ). To 
characterize tolerance, three effective concentrations for the microbial response to drilling fluid load were assessed: (i) the minimum suppressive concentration (MSC) of drilling fluid, which reduces microbial growth rate compared to control drilling fluid-free media; (ii) the Minimum Inhibitory Concentration (MIC) corresponding to the minimum drilling fluid concentration needed to stop microbial growth; and (iii) the Minimum Bactericidal Concentration (MBC) as a minimum concentration of drilling fluid resulting in $99.9 \%$ microbial population kill. Figure 1 represents the plot of microbial growth dynamics as a function of drilling fluid load with points used for calculation of effective concentrations of drilling fluids.

\section{Microbial growth capacity and viability in drilling fluids}

The mineral media supplemented with drilling fluids at concentrations ranging from 0.1 to $10 \%$ of oil with two-fold increment were seeded with a microorganism at concentration of $10^{5} \mathrm{cell} / \mathrm{ml}$ and incubated at $25^{\circ} \mathrm{C}$ with agitation at $100 \mathrm{rpm}$ for 7 days. Microbial suspensions were then serially diluted in phosphate buffer saline and plated on agar basic media with glucose to determine the number of viable cells after treatment. The cell number was represented as log $\mathrm{CFU} / \mathrm{ml}$. The growth capacity was characterized by the increase in cell number as a function of oil concentration in the media. Two parameters characterized the growth capacity: (i) the peak concentration (Cp) as the concentration of drilling fluid, which provides maximum growth rate, and (ii) the minimum inhibitory concentration (MIC) corresponding to the minimum drilling fluid concentration needed to stop microbial growth. To avoid confusion, the minimum inhibitory concentration in this assay was labeled as MIC-2. Viability of microbial cells in response to a drilling fluid was characterized by the minimum bactericidal concentration (MBC) as a minimum concentration of drilling fluid resulting in $99.9 \%$ microbial population kill. In this assay, the minimum bactericidal concentration was labeled as MBC-2.

\section{Hydrocarbon degradation in drilling fluids}

Microbial cultures of $10^{5} \mathrm{CFU} / \mathrm{ml}$ were added to $250 \mathrm{ml}$ Erlenmeyer flasks containing $50 \mathrm{ml}$ of mineral media and one of the drilling fluids at peak concentration. The cultures were incubated at $25^{\circ} \mathrm{C}$ with agitation at $100 \mathrm{rpm}$ for 7 days. The uninoculated media with drilling fluids served as controls. After 7 days, samples were aseptically taken for viable cell number and total petroleum hydrocarbons (TPH). The viable cell number was determined after growth of serially diluted samples on agar basic media with glucose. Growth rate was determined as the difference between the initial cell number in the population and the cell number after exposure to the drilling fluid $(\Delta \log \mathrm{CFU} / \mathrm{ml})$.

The initial and residual TPH were extracted with $\mathrm{CCl}_{4}$ from the liquid cultures and analyzed by FT-IR after passing the extract through a Florisil column. The extract was quantitatively measured after calibration with a standard mixture $(\mathrm{v} / \mathrm{v})$ of $\mathrm{n}$-hexadecane $(37.5 \%)$, isooctane $(37.5 \%)$, and benzene (25\%). The spectrum was recorded between the $3,100-2,800 \mathrm{~cm}^{-1}$ range. The absorbance values were measured at 2,926 $\mathrm{cm}^{-1}$ with an IR spectrophotometer (UNICAM SP1000, UK). The TPH content was related to the $\mathrm{CH}_{2}$ group number [17].

\section{Results}

\section{Microbial tolerance to drilling fluids}

The microbial tolerance is the ability of microorganisms to survive and remain active when exposed to a toxic substrate. High tolerance is beneficial for microbial degradation of drilling fluid waste at relatively high hydrocarbon concentrations, thereby reducing the need for extensive waste dilution prior to bioremediation. Two microorganisms with proved hydrocarbon-degrading potential, $R$. picketti BP20 and A. piechaudii KN1were used in the present study. Plaza and coauthors reported that co-culture of these strains removed $91 \%$ of TPH in oilcontaminated waste after 30 days incubation [17]. The initial TPH load in cultivation/degradation media was $1.9 \%$. The present study investigated the possibility and conditions to increase oil load without impairing biodegradation rate. R. picketti $\mathrm{BP} 20$ and A. piechaudii $\mathrm{KN} 1$ demonstrated different tolerance levels depending on the drilling fluid and co-substrate types. The easily consumable co-substrate (either glucose or pyruvate) was used to avoid ambiguity in distinguishing between starvation and the toxic effects of the drilling fluids. The dynamics of microbial growth on easily-consumable substrate in response to the addition of the drilling fluid followed this scenario: a microorganism grew in the presence of relatively low concentrations of a drilling fluid at growth rate typical for those on easily consumable substrate, i.e., the drilling fluid had no impact on microbial growth rate until toxic level was reached. The first sign of toxic effect can be associated with the suppression of the microbial growth. The minimum concentration causing growth rate reduction compared to drillingfluid-free media defines the Minimum Suppressive Concentration (MSC). Increasing drilling fluid concentrations further reduced microbial metabolic activity until microbial growth completely stopped at the Minimum Inhibitory Concentration (MIC) of drilling fluid. The drilling fluids at the concentrations above MIC level killed microbial cells. The killing of $99.9 \%$ of the microbial population by the drilling fluid was defined as the Minimum Bactericidal Concentration (MBC).

MSC for R. picketti BP20 incubated in the presence of glucose and DF was around 1\% diesel (Figure 1). Emulsification of water in diesel (DIE) reduced MSC values to $0.8 \%$; while diesel emulsification in water (DDE) caused microbial growth suppression only at $2 \%$ diesel. The replacement of diesel with low aromatic oil improved microbial tolerance. LAF had no impact on R. picketti BP20 growth up to $1.3 \%$ oil. Emulsification of low aromatic oil increased MSC values to $2.5 \%$ for LAIE and $4.2 \%$ for LADE.

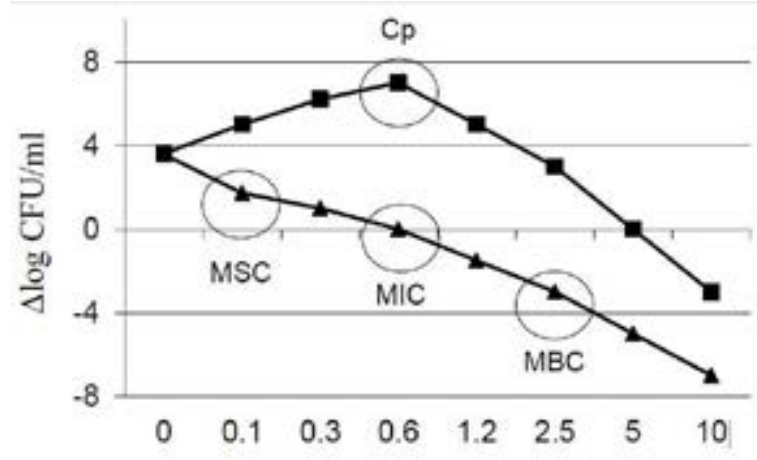

Drilling fluid concentration $(\%)$

Figure 1: Dynamics of the microbial growth as a function of oil concentration in the presence of co-substrate and drilling fluid $(\boldsymbol{\Delta})$ and in the media containing only drilling fluid( $\mathbf{})$. Cp: the peak concentration as the concentration of drilling fluid, which provides maximum growth rate: MSC: the minimum suppressive concentration of drilling fluid, which reduces microbial growth rate compared to control drilling fluid-free media; MIC: the minimum inhibitory concentration (MIC) corresponding to the minimum drilling fluid concentration needed to stop microbial growth; MBC: the minimum bactericidal concentration (MBC) as a minimum concentration of drilling fluid resulting in $99.9 \%$ microbial population kill. 
In the presence of pyruvate, $R$. picketti BP20 response to diesel drilling fluids followed similar trends, but effective concentrations were a little bit lower than in glucose-containing media: MSC were below $0.8 \%$ for DF, $0.6 \%$ for DIE and $1 \%$ for DDE. However, MSC values for low aromatic fluid and emulsions in pyruvate media were higher than corresponding values in the presence of glucose: $2.5 \%$ for LAF, $3.3 \%$ for LAIE and 5\% for LADE.

A. piechaudii KN1 demonstrated higher tolerance than $R$. picketti BP20 under all tested conditions. In the presence of glucose, MSC values for DF, DIE and DDE were $2.1 \%, 1.3 \%$ and $4.2 \%$, respectively (Figure 2). With low aromatic fluid and emulsions A. piechaudii KN1 showed even higher tolerance: $3.3 \%$ for LAF; $4.2 \%$ for LAIE and $5 \%$ for LADE. In the presence of pyruvate, the tolerance of $A$. piechaudii $\mathrm{KN} 1$ was higher than in glucose media for both diesel and low aromatic drilling fluids: $3.3 \%$ for DF; $2.1 \%$ for DIE; $5 \%$ for DDE; $4.2 \%$ for LAF; $5 \%$ for LAIE; and $6.7 \%$ for LADE.

The impact of drilling fluids on the viability of the hydrocarbondegrading microorganisms was quantified by the minimum inhibitory (MIC) and bactericidal (MBC) concentrations. Similarly to the impact of drilling fluids on the microbial growth rate, viability was enhanced when low aromatic oil replaced diesel. Invert emulsions reduced, while direct emulsion enhanced diesel concentrations which affect microbial viability. The microbial viability in response to low aromatic fluids increased in the order: non-emulsifier oil $<$ invert emulsion $<$ direct emulsion. The tolerance of $A$. piechaudii $\mathrm{KN} 1$ was higher than that of R. picketti BP20 to all tested drilling fluids.

The growth of $R$. picketti BP20 was inhibited at levels as low as 1.7 $-3.3 \%$ of diesel based drilling fluids and $2.5-6.7 \%$ of low aromatic

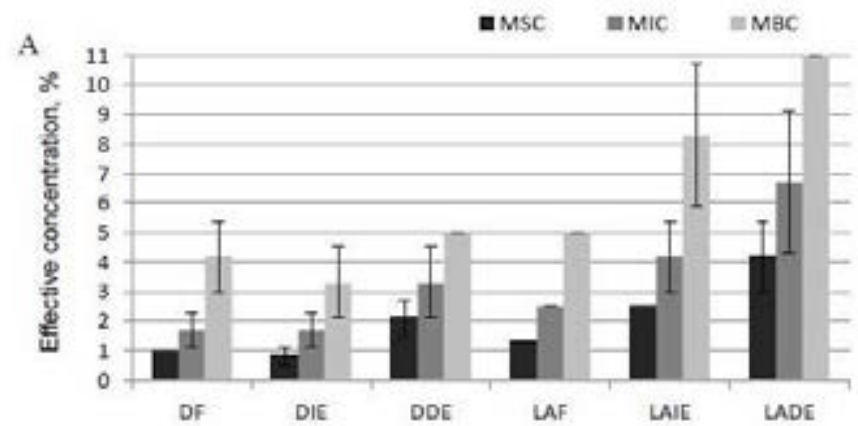

B

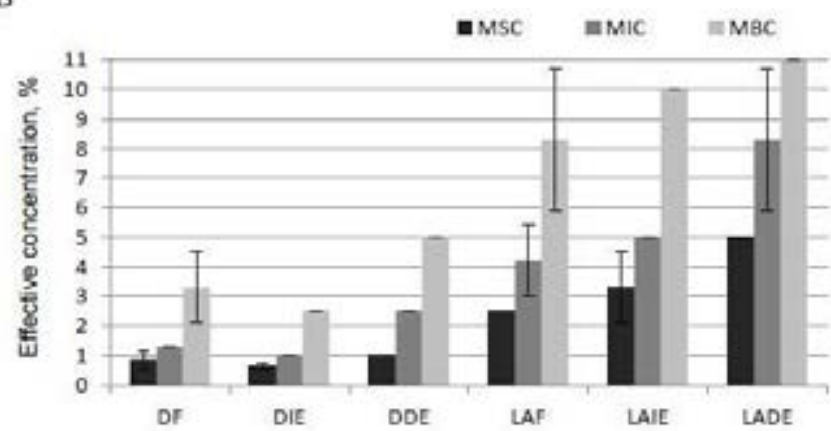

Figure 2: Effective concentrations (MBC, MIC and MSC) observed for $R$ picketti BP20 incubated in the presence of drilling fluids: Diesel based Fluid (DF), Diesel Invert Emulsion (DIE), Diesel Direct Emulsion (DDE), Low Aromatic Fluid (LAF), Low Aromatic Invert Emulsion (LAIE) and Low Aromatic Direct Emulsion (LADE), and co-substrate: glucose (A) and sodium pyruvate (B). drilling fluids in the presence of glucose (Figure 3). In the presence of pyruvate, $R$. picketti BP20 growth stopped at nearly twice lower concentrations of diesel fluids and nearly twice higher concentration of low aromatic oil. Similarly, MBC values were around 1.5 times higher in glucose media than in the presence of pyruvate for diesel fluids: 3.3 $-5 \%$ and $2.5-3.3 \%$, respectively; but, 1.5 times lower for low aromatic fluids: 5 - > $10 \%$ and $8.3->10 \%$, respectively.

In contrast, $A$. piechaudii $\mathrm{KN} 1$ tolerance was higher in the presence of pyruvate than in glucose media for both tested oil types (Figure 3). The inhibition of $A$. piechaudii $\mathrm{KN} 1$ growth was observed at $3.3-8.3 \%$ for diesel drilling fluids and at $5-10 \%$ for low aromatic drilling fluids in the presence of glucose, but, $5-10 \%$ and $8.3-10 \%$, respectively, in the presence of pyruvate. MBC values of diesel increased from $6.7-10 \%$ in the presence of glucose to $8.3->10 \%$ in the presence of pyruvate. For low aromatic fluid under all tested conditions, $\mathrm{MBC}$ values against $A$. piechaudii $\mathrm{KN} 1$ were more than $10 \%$ (maximum tested concentration).

\section{Hydrocarbon assimilation as a function of the oil concentration}

To assess the microbial ability to assimilate hydrocarbons from drilling fluids, the drilling fluids were added to mineral media as sole carbon and energy source at oil concentrations ranging from $0.1 \%$ to $10 \%$ with two-fold increment. After 7 day incubation, changes in microbial population were assessed for each oil concentration. In general, drilling fluid addition induced microbial growth until it reached maximum values at the peak oil concentration $(\mathrm{Cp})$. Further increase of oil concentration caused microbial growth reduction until complete growth inhibition which was observed at the minimum inhibitory concentration (MIC-2) of oil followed by the reduction of microbial cell number. $99.9 \%$ killing of the microbial population was achieved at the minimum bactericidal concentration (MBC-2).

For both microorganisms, the MBC-2 values were equal or higher than $\mathrm{MBC}$ values obtained in the presence of co-substrates (Figure 4). The MIC-2 values of DF against $R$. picketti BP20 were $4-5$ times lower than corresponding MIC values in glucose and pyruvate media. Similarly, MIC-2 values of DF against A. piechaudii KN1 were $3-4$ times lower than corresponding MIC values. The peak concentrations of most tested drilling fluids correlated well with corresponding MSC values. Except for DF media, Cp values were $1.5-2.5$ times lower than corresponding MSCs, which indicates that the maximum (peak) growth rate was observed one step (according to two-fold increment) before growth suppression. For DF media, Cp values were around 5 times and 10 times lower than the corresponding MSC values against R. picketti $\mathrm{BP} 20$ and $A$. piechaudii $\mathrm{KN} 1$, respectively.

At $\mathrm{Cp}$ values, drilling fluids provided the highest growth rates. The lowest growth rates and corresponding TPH (total petroleum hydrocarbon) removal were observed for DF: 3 - 7\% hydrocarbon reduction resulted in biomass increase of $0.8-1.2 \mathrm{log} \mathrm{CFU} / \mathrm{ml}$ (Figure 5). Emulsification significantly improved metabolic ability of microbial cultures. $11-12 \%$ diesel was assimilated by microbial populations from DIE which increased microbial cell number by $2.1 \log$ CFU/ $\mathrm{ml}$. In the presence of DDE, microbial population grew by $2.8-3.1$ $\log \mathrm{CFU} / \mathrm{ml}$ and assimilated $19-21 \%$ diesel. While the hydrocarbondegrading capacity against diesel fluid and emulsions were similar for both microbial cultures, A. piechaudii $\mathrm{KN} 1$ demonstrated higher metabolic potential against low aromatic drilling fluid than $R$. picketti BP20. Both strains assimilated low aromatic drilling fluid with higher rates than corresponding diesel based fluids, which resulted in higher growth rates. And again, the degradation potential and growth rates for 
Citation: Kostenko V, Martinuzzi RJ, Hareland G (2013) Impact of Oil-Based Drilling Fluids Emulsification on Tolerance and Hydrocarbon-Degrading Potential of Ralstonia pickettii and Alcaligenes piechandii. J Pet Environ Biotechnol 4: 140. doi:10.4172/2161-1459.1000140

both strains increased in the order: LAF < LAIE < LADE. In particular, A. piechaudii $\mathrm{KN} 1$ assimilated $27 \%, 39 \%$ and $46 \%$ of low aromatic oil with corresponding growth rates of $3.8 \log \mathrm{CFU} / \mathrm{ml}, 4.4 \log \mathrm{CFU} / \mathrm{ml}$ and $5.2 \log \mathrm{CFU} / \mathrm{ml}$, respectively. R. picketti BP20 assimilated $19 \%$, $31 \%$ and $49 \%$ of low aromatic oil, respectively, that resulted in $3.1-4.3$ $\log \mathrm{CFU} / \mathrm{ml}$ growth rates.

\section{Discussion}

Based on the aforementioned observations, the response of hydrocarbon-degrading microorganisms to a variety of drilling fluids is characterized by microbial growth at relatively low concentrations of drilling fluid; followed by growth suppression and further microbial cell killing at higher drilling fluid concentrations. The critical level showing the switch between microbial growth stimulation and suppression is characterized by microbial tolerance. The higher tolerancelevel indicates that a microorganism is active (e.g., degrade hydrocarbons) at higher concentrations of hydrocarbons, which gives a marked advantage in the treatment of hydrocarbon contaminated waste, e.g., drilling waste. As previously reported, hydrocarbon degradation by microorganisms was observed at hydrocarbon concentrations as low as 1\% [9-11]. Low acceptable (i.e., allowing growth) levels of hydrocarbons imply that the bioremediation process requires extensive dilution of the waste, which reduces the bioremediation economical feasibility. Thus, enhancement of microbial tolerance to hydrocarbon increases the success potential in designing effective bioremediation technologies.

As demonstrated in the present study, microbial tolerance to drilling fluids is a complex issue which is attributed in part to the inherent microbial properties, but also to properties of the substrate
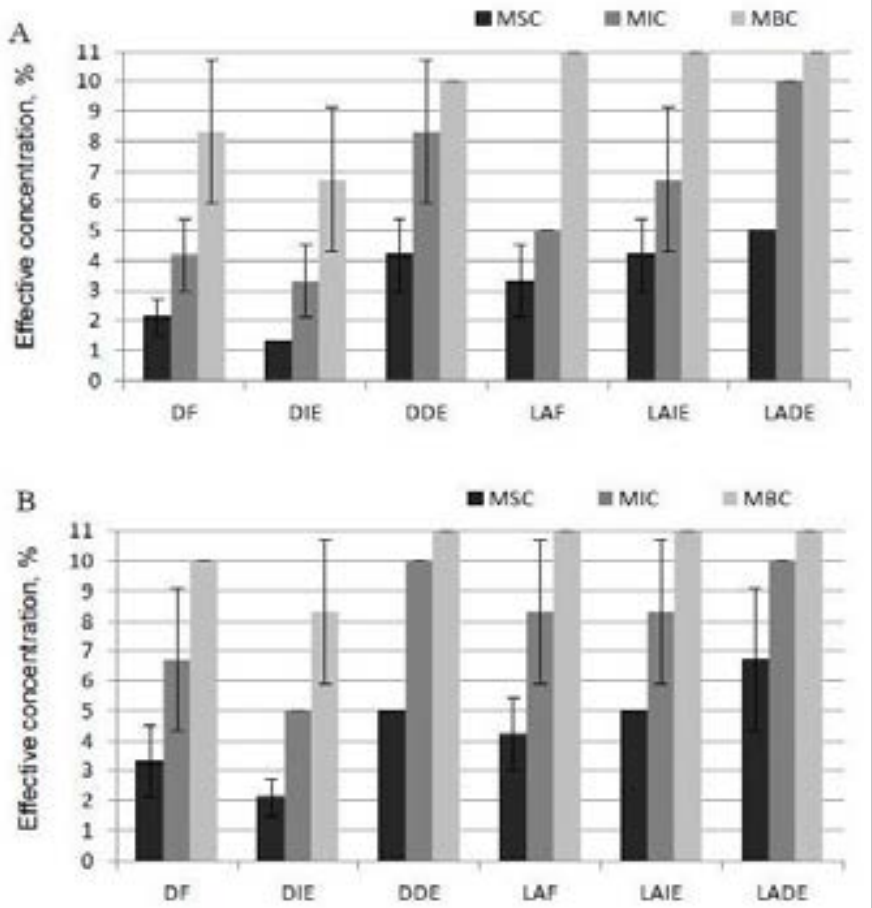

Figure 3: Effective concentrations (MSC, MIC and MBC) observed for $A$. piechaudii KN1 incubated in the presence of drilling fluids: Diesel based Fluid (DF), Diesel Invert Emulsion (DIE), Diesel Direct Emulsion (DDE), Low Aromatic Fluid (LAF), Low Aromatic Invert Emulsion (LAIE) and Low Aromatic Direct Emulsion (LADE), and co-substrate: glucose (A) and sodium pyruvate (B).

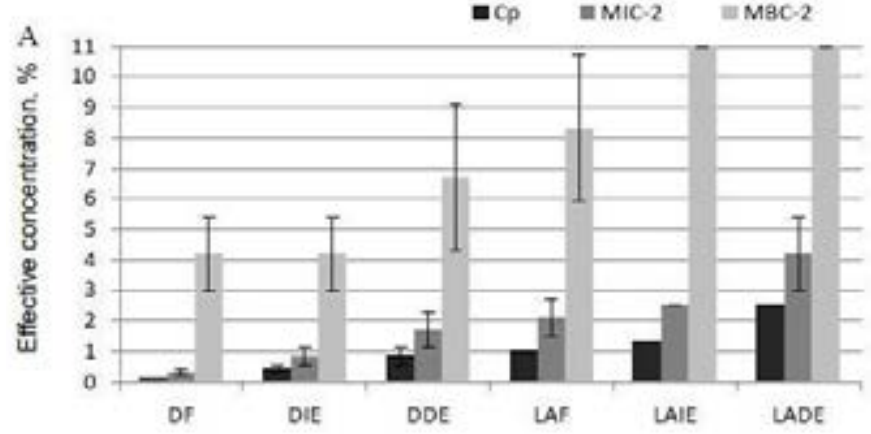

B

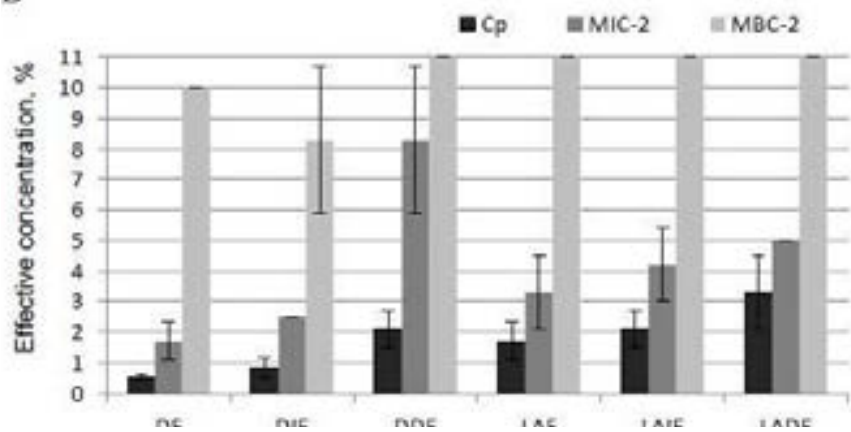

Figure 4: Effective concentrations (Cp, MIC-2 and MBC-2) observed for $R$. picketti BP20 (A) and $A$. piechaudii KN1(B) incubated in the presence of drilling fluids: Diesel based Fluid (DF), Diesel Invert Emulsion (DIE), Diesel Direct Emulsion (DDE), Low Aromatic Fluid (LAF), Low Aromatic Invert Emulsion (LAIE) and Low Aromatic Direct Emulsion (LADE).
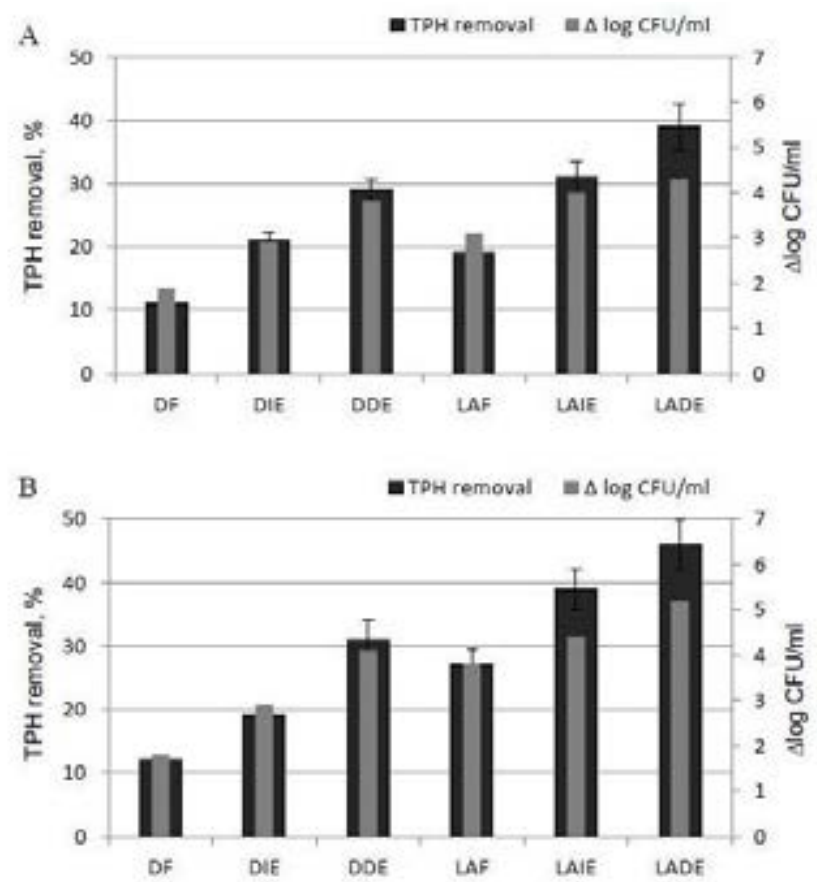

Figure 5: Total Petroleum Hydrocarbon (TPH) removal and corresponding microbial growth ( $\Delta$ log CFU/ml) observed for $R$. picketti BP20 (A) and $A$ piechaudii $\mathrm{KN} 1(\mathrm{~B})$ incubated in the presence of drilling fluids: Diesel based Fluid (DF), Diesel Invert Emulsion (DIE), Diesel Direct Emulsion (DDE), Low Aromatic Fluid (LAF), Low Aromatic Invert Emulsion (LAIE) and Low Aromatic Direct Emulsion (LADE). 
and cultivation conditions. Microorganisms tested in the present study demonstrated different levels of inherent tolerance to drilling fluids. A. piechaudii KN1demonstrated higher tolerance levels under all tested conditions compared to $R$. picketti BP20 response. Nevertheless, the impact of different drilling fluids on the viability and productivity of both microbial strains had similar trends. Microbial growth and TPH degradation rates increased when diesel was replaced with low aromatic oil. Further improvement was achieved via emulsification. Moreover, higher productivity was observed in direct $(\mathrm{O} / \mathrm{W})$ emulsions than in invert (W/O) emulsions. Similarly, viability of microorganisms in low aromatic fluids and emulsions was higher than in corresponding diesel drilling fluids. Tolerance to low aromatic fluids increased in the sequence: non-emulsifier oil < invert emulsion < direct emulsion. However, for diesel based drilling fluids, direct emulsion enhanced, but invert emulsions reduced microbial viability when compared to nonemulsified oil

The tolerance mechanisms for the tested microorganisms are poorly understood. It is not in the scope of the present study to explain the genetic mechanisms of tolerance to hydrocarbons, but rather to understand the microbial tolerance regulation by substrate conditions, in particular, emulsification (direct and invert). Emulsification and the action of surfactants increase bioavailability of the poorly soluble hydrocarbons that may improve hydrocarbons uptake and intracellular metabolism on the one hand and hydrocarbon toxicity to the microbial cells on the other hand. In particular, the involvement of intracellular hydrocarbon-metabolizing enzymes implies that the substrates must enter the cell prior to being metabolized. The poorly soluble compounds such as hydrocarbons have to be solubilized to enter cells for bioremediation reactions. However, increased solubility and transfer rate of hydrocarbon may impact not only metabolic processes, but also promotes the detrimental interaction of hydrocarbons with microbial cell membranes and functional molecules that result in cell deactivation or death. The present study demonstrated that emulsification/dispersion and solubilization of diesel and low aromatic oil allowed better assimilation of these substrates. The TPH removal increased from $11-12 \%$ for non-emulsified diesel to $19-21 \%$ for diesel invert emulsion, and to $29-31 \%$ for diesel direct emulsion. Similarly, assimilation of $19-27 \%$ of oil from non-emulsified low-aromatic oil drilling fluid increased to $31-46 \%$ in low aromatic emulsions. The emulsification also increased effective concentrations of drilling fluids that allow higher load of hydrocarbons in waste to be acceptable for biodegradation. While maximum productivity was observed at $0.1-$ $0.5 \%$ of diesel and $1.0-1.5 \%$ of low aromatic oil, the emulsification of oil in aqueous phase allowed assimilation of $1.0-2.0 \%$ of diesel and 2.5 $-4.0 \%$ of low aromatic oil. On the other hand, emulsification of water in diesel resulted in reduced viability of the tested microorganisms compared to the incubation in non-emulsified diesel or diesel direct emulsions. However, either invert or direct emulsions of low aromatic oil provided better viability of microorganisms compared to nonemulsified oil. Thus, the biodegrading potential and the tolerance of microorganisms are functions of: substrate chemical (diesel vs. low aromatic oil) and physical (non-emulsified, invert or direct emulsions) state; and concentration of oil in cultivation media.

Another factor responsible for microbe-hydrocarbon interaction is the surface area between the organic phase and the aqueous phase. Microorganisms have been shown to adhere and grow on the surface of the hydrocarbon droplets dispersed in water. In invert emulsions, microorganisms were also able to attach to the oil-water interface, but grew only in water droplets [7,18]. Thus, the type of emulsion may determine the ability of microorganisms to grow and degrade hydrocarbons in oil-water biphasic systems. In direct emulsions, microbial cells move freely in the continuous water phase and have free access to oxygen and nutrients, including hydrocarbons dispersed in water, providing high growth rates and reducing toxic effect of hydrocarbon encapsulated in droplets. In contrast, invert emulsions are water droplets in the continuous oil phase which confine microbial growth within the water droplets, since the continuous oil phase cannot sustain microbial growth, even being the sole carbon source. Moreover, bulk oil can limit microbial growth and activity in water droplets by reducing: bioavailability of oxygen, mass transfer of nutrients, and solubility of hydrocarbon as main substrate [8]. The microbe interaction with bulk oil also increases hydrocarbon toxicity against microorganisms. Thus, direct emulsions of oil, either toxic or not, have a potential to enhance microbial ability to assimilate and resist to hydrocarbon substrate such as drilling fluids.

In the present study, the emulsions were added to cultivation media. The increased water-cut is intended to break emulsions. However, agitation allowed keeping small oil droplets dispersed in media for direct emulsions, and let bulk oil from invert emulsions to form large droplets in media (data not shown). This conversion represents the biological treatment where cultivation media/fertilizers are added to facilitate microbial activity, and/or waste is diluted to avoid the toxic effects of hydrocarbons. The increased amount of aqueous phase (in our case, cultivation media) and oil dispersion (both small and large droplets) improved conditions for microbial growth and activity. However, the level of dispersion (small vs. large droplets) had a different impact on microbial activity. The microbial growth and TPH degradation were higher in direct-emulsion-containing media providing good oil dispersion than in media supplemented with invert emulsions producing large droplets. Similarly, addition of nonemulsified oils in culture media resulted in large oil droplets dispensed in the media (data not shown). However, microbial activity under these conditions was lower than in media supplemented with emulsions, which indicate that surfactants contribute to microbial assimilation of the hydrocarbons from drilling fluids. These trends were observed for both diesel and low aromatic oil. In contrast, microbial viability as a function of drilling fluid states depended on the substrate toxicity. For the non-toxic substrate (low aromatic oil), both minimization of oil droplet size and oil solubilization improved microbial tolerance. However, for the toxic substrate (diesel), minimization of oil droplets size increased microbial tolerance, but oil solubilization reduced it.

Along with the oil phase, surfactants and Hydrophilic-Lipophilic Balance (HLB) values may have toxic effects on microbial activity by interfering with microbial cell membranes and through functional molecules deactivation. The Span-Tween surfactant system at HLB ranging from 4 to 14 systems used in the present study had no impact on the growth of $A$. piechaudii $\mathrm{KN} 1$ and $R$. picketti BP20 (data not shown). Bruneim et al. [19] supported our observation that Span 80 and Tween 80 had no negative impact on microbial growth, but improved hydrocarbon degradation via emulsification [19].

The tolerance of the hydrocarbon-degrading microorganisms also responded to the addition of a co-substrate. The co-substrate (either glucose or sodium pyruvate) had a different impact on microbial growth and viability in response to drilling fluids. Glucose provided higher tolerance of $R$. picketti BP20 to diesel, but pyruvate enhanced tolerance to low aromatic oil. The tolerance of $A$. piechaudii KN1 was better supported by pyruvate against both diesel and low aromatic oil. Thus, co-substrates may improve hydrocarbon-containing waste degradation, but can be also used for predicting microbial tolerance, 
and hence the potential for degradation of high concentration of drilling fluid in waste after short term exposure (e.g., a 24 hour cultivation as in the present study). The assimilation of hydrocarbon is usually a slow process. It may take more than a week to get accurate data on microbial tolerance and productivity at different hydrocarbon concentrations when the hydrocarbon is a sole carbon and energy source. However, an easily-consumable co-substrate (e.g., glucose and pyruvate) allows quick microbial growth, which is, however, regulated by the drilling fluid supplied. The present study demonstrated that $\mathrm{MBC}$ and $\mathrm{Cp}$ values obtained after 7-day exposure to the drilling fluids correlated well with corresponding $\mathrm{MBC}$ and MSC values obtained after 24 hour exposure to the drilling fluid with the co-substrate. In contrast, MIC values were significantly lower in the absence of co-substrates indicating that the co-substrate supports microbial growth in the presence of drilling fluid and provides higher numbers of microbial cells ready for hydrocarbon assimilation. Therefore, MBC and MSC values may be used as effective diagnostic parameters for predicting viability and productivity of hydrocarbon-degrading microorganisms and possible acceptable hydrocarbon load for the biodegradation process; while MIC values allowed a selection of the most effective co-substrate system.

Using co-substrate-containing and co-substrate-free models in the present study shows that the best hydrocarbon degradation (for the tested conditions) may be achieved by assimilation of low aromatic direct emulsion by $A$. piechaudii $\mathrm{KN} 1$, and sodium pyruvate may improve degradation potential of this microorganism. In this case, $46 \%$ of TPH was removed from drilling fluid waste after a 7-day exposure to microbial culture with initial oil load of $3.5 \%$. The pyruvate may increase optimum oil load up to $5 \%$, and hence improve waste cleaning.

Thus, the main observation from this study identifies the parameters to improve hydrocarbon-contaminated waste treatment: (i) type of microorganism; (ii) type and state of substrate; and (iii) co-substrate. The essential element is microorganisms or microbial consortia with high hydrocarbon-degrading potential and high tolerance to hydrocarbon. While hydrocarbon-degrading potential provides a high metabolic rate, high tolerance allows for an increase in hydrocarbon load in cultivation media or higher concentration of hydrocarbon in waste to be degraded without microbial growth impairment. Another important element is the toxicity and biodegradability of substrate. As demonstrated in the present paper and as previously reported, low aromatic oil had lower toxicity and higher biodegradability than diesel. So, replacement of diesel with low aromatic mineral and synthetic oil is a promising step in the improvement of drilling waste treatment. However, both diesel and low aromatic oil utilization may be improved by emulsification. The highest biodegradation potential and the lowest toxicity were determined for direct $(\mathrm{O} / \mathrm{W})$ emulsions. Thus, the development of technologies where bulk oil from waste is emulsified in water (and/or invert emulsions are reversed to direct emulsions) may improve waste degradation. The improvement of hydrocarbon waste degradation may be also be achieved by setting up reinforcing cultivation conditions, e.g., selecting an appropriate co-substrate.

\section{Acknowledgement}

This work was supported by NSERC Industrial Research Chair Awards to G.H., NSERC RTI Program to RJM and Talisman Energy Inc.

\section{References}

1. Sachez G, Leon N, Esclapes M, Galindo I, Martinez A, et al. (1999) Environmentally Safe Oil-Based Fluids for Drilling Activities. SPE/EPA Exploration and Production Environmental Conference, 1-3 March Austin Texas USA.

2. Melton HR, Smith JP, Mairs HL, Bernier RF, Garland E, et al. (2004) Environmental aspects of the use and disposal of non aqueous drilling fluids associated with offshore oil \& gas operations. SPE International Conference on Health, Safety, and Environment in Oil and Gas Exploration and Production, 29-31 March Calgary, Alberta, Canada.

3. Ward O, Singh A, Van Hamme J (2003) Accelerated biodegradation of petroleum hydrocarbon waste. J Ind Microbiol Biot 30: 260-270.

4. Atla RM (1991) Microbial Hydrocarbon Degradation-Bioremediation of Oil Spills. J Chem Tech Biotechnol 52: 149-156

5. Oberbremer A, Muller-Hurtig R, Wagner F (1990) Effect of the addition of microbial surfactants on hydrocarbon degradation in a soil population in a stirred reactor. Appl Microbiol Biot 32: 485-489.

6. Tiehm A (1994) Degradation of polycyclic aromatic hydrocarbons in the presence of synthetic surfactants. Appl Environ Microbiol 60: 258-263.

7. Coty VF, Gorring RL, Heilweil IJ, Leavitt RI, Srinivasan S (1971) Growth of microbes in an oil-continuous environment. Biotechnol Bioeng 13: 825-842.

8. Sikkema J, de Bont JA, Poolman B (1995) Mechanisms of membrane toxicity of hydrocarbons. Microbiol Rev 59: 201-222.

9. Raymond RL, Jamison VW, Hudson JO (1967) Microbial hydrocarbon cooxidation. I. Oxidation of mono- and dicyclic hydrocarbons by soil isolates of the genus Nocardia. Appl Microbiol 15: 857-865.

10. Worsey MJ, Williams PA (1975) Metabolism of toluene and xylenes by Pseudomonas (putida (arvilla) mt-2: evidence for a new function of the TOL plasmid. J Bacteriol 124: 7-13.

11. Raghavan TM, Furtado I (2000) Tolerance of an estuarine halophilic archaebacterium to crude oil and constituent hydrocarbons. Bull Environ Contam Toxicol 65: 725-731.

12. Margesin R, Hämmerle M, Tscherko D (2007) Microbial activity and community composition during bioremediation of diesel-oil-contaminated soil: effects of hydrocarbon concentration, fertilizers, and incubation time. Microb Ecol 53 259-269.

13. Cerniglia CE, Freeman JP, Althaus JR, van Baalen C (1983) Metabolism and toxicity of 1- and 2-methylnaphthalene and their derivatives in cyanobacteria. Arch Microbiol 136: 177-183.

14. Calder JA, Lader JH (1976) Effect of dissolved aromatic hydrocarbons on the growth of marine bacteria in batch culture. Appl Environ Microbiol 32: 95-101.

15. Singh M, Dasai JD (1986) Uptake water insoluble substrates by microorganisms J Sci Ind Res 45: 413-417.

16. Leahy JG, Colwell RR (1990) Microbial degradation of hydrocarbons in the environment. Microbiol Rev 54: 305-315.

17. Płaza GA, Jangid K, Lukasik K, Nałecz-Jawecki G, Berry CJ, et al. (2008) Reduction of petroleum hydrocarbons and toxicity in refinery wastewater by bioremediation. Bull Environ Contam Toxicol 81: 329-333.

18. Nadarajah N, Singh A, Ward OP (2002) De-emulsification of petroleum oil emulsion by a mixed bacterial culture. Process Biochem 37: 1135-1141.

19. Bruheim P, Bredholt H, Eimhjellen K (1999) Effects of surfactant mixtures including Corexit 9527, on bacterial oxidation of acetate and alkanes in crude oil. Appl Environ Microbiol 65: 1658-1661. 